\title{
Linx
}

Revue des linguistes de l'université Paris X Nanterre

$54 \mid 2006$

La cause : approche pluridisciplinaire

\section{Verbes causatifs, discours causaux et coréférence événementielle}

Laurence Danlos

\section{(2) OpenEdition}

\section{Journals}

Édition électronique

URL : http://journals.openedition.org/linx/535

DOI : $10.4000 /$ linx.535

ISSN : 2118-9692

Éditeur

Presses universitaires de Paris Nanterre

\section{Édition imprimée}

Date de publication : 1 juin 2006

Pagination : 233-246

ISSN : 0246-8743

\section{Référence électronique}

Laurence Danlos, «Verbes causatifs, discours causaux et coréférence événementielle », Linx [En ligne], 54 | 2006, mis en ligne le 01 août 2007, consulté le 19 avril 2019. URL : http:// journals.openedition.org/linx/535; DOI : 10.4000/linx.535 


\section{Verbes causatifs, discours causaux et coréférence événementielle}

\section{Laurence Danlos}

\section{Université Paris 7, IUF, LATTICE}

\section{Introduction}

Nous nous intéressons aux discours causaux comprenant deux phrases, l'une décrivant la cause, l'autre décrivant l'effet par le truchement d'un verbe causatifi'. On peut distinguer d'une part les verbes causatifs psychologiques (e.g. agacer, amuser) qui admettent un sujet référent soit à un événement (Cet événement a agacé Marie), soit à un fait (Le fait) que Fred ne soit pas venu a agacé Marie), soit à une entité (Fred / cette chaise a agacé Marie), d'autre part les verbes causatifs non psychologiques (fêler, casser) qui n'admettent généralement qu'un sujet référent à une entité (Fred a félé la carafe, *Cet incident a felé la carafe) $)^{2}$.

Selon l'ordre dans lequel apparaissent les phrases, et selon la nature du verbe causatif et de son sujet, on observe les paradigmes suivants.

(1) a Fred a heurté la carafe contre l'évier. Cela / Cet incident a agacé Marie.

b Fred a heurté la carafe contre l'évier. Il a agacé Marie.

c Fred a agacé Marie. Il a heurté la carafe contre l'évier.

\footnotetext{
${ }^{1}$ Cet article est une adaptation française et mise à jour de (Danlos 2001a).

2 Il existe cependant des verbes causatifs non psychologiques qui admettent un sujet événementiel : Cet accident d'avion a tué 44 personnes. Nous n'examinerons pas ces cas.
} 
(2) a \#Fred a beurté la carafe contre l'évier. Cela/ cet incident l'a fêlée 3 .

b Fred a heurté la carafe contre l'évier. Il l'a fêlée.

c Fred a fêlé la carafe. Il l'a beurtée contre l'évier.

En (1a), le pronom cela ou le GN anaphorique cet incident réfère à l'événement décrit dans la première phrase. Une telle relation de coréférence événementielle ne peut s'observer en (2a) à cause des contraintes imposées par le verbe fêler sur son sujet ${ }^{4}$.

Les discours (b) sont obtenus à partir des discours (a) en remplaçant le sujet événementiel par le sujet il référent à l'entité Fred, agent de la cause. La paire (1a)-(1b) réunit tous les ingrédients pour parler de métonymie : en (b), l'agent de la cause est employée métonymiquement à la place de la cause. Cela nous amène à dire qu'un verbe causatif non-psychologique comme féler n'admet qu'un sujet métonymique. Cette analyse s'accorde en tout point avec la structure événementielle et la structure de qualia des verbes causatifs proposées dans le cadre du Lexique Génératif (Pustejovsky 1995). Cette décomposition lexicale pour les verbes causatifs sera résumée dans la Section 2.1. Elle nous permettra de poser que les discours (b) mettent en jeu une relation de coréférence événementielle comme c'est explicitement le cas en (1a).

Les discours (c) sont obtenus à partir des discours (b) en inversant l'ordre des phrases (et en inversant les relations anaphoriques). Nous montrerons qu'ils mettent aussi en jeu une relation de coréférence événementielle, (Section 2.2).

Ces analyses par coréférence événementielle pour les discours (b) et (c) n'impliquent pas cependant que ces discours soient parfaitement symétriques. La paire en (3) met en évidence un contraste entre ces deux types de discours.

(3) b \#Fred a heurté la carafe contre l'évier. Il l'a fellée à midi'.

c Fred a fêlé la carafe à midi. Il l'a heurtée contre l'évier.

Les exemples (3b) et (3c) sont obtenus respectivement à partir de (2b) et (2c) en ajoutant l'adverbe temporel à midi qui vient modifier le verbe causatif fêler. On constate que (3b) ne reçoit plus d'interprétation causale contrairement à (3c) : si l'on accepte (3b) c'est dans une interprétation où les événements de heurtage et de fêlage sont considérés comme indépendants, sans lien de cause à effet. Nous expliquerons dans la Section 3 le contraste en (3) en introduisant un typage des relations de coréférence (qui est valide tant pour les événements que pour les entités).

Cette étude sera aussi l'occasion de préciser différentes propriétés des verbes causatifs.

\footnotetext{
${ }^{3}$ Le symbole * préfixe un discours mal formé.

${ }^{4}$ Dans un style relâché oral, le discours obtenu en remplaçant cela par ça passe : Fred a heurté la carafe contre l'évier. Ça l'a fêlée.

${ }^{5}$ Par convention dans cet article, tout discours qui n'a pas d'interprétation causale est précédé du signe \#.
} 


\section{Structure événementielle et analyse par coréférence événementielle}

\subsection{Structure événementielle des verbes causatifs}

La structure événementielle de Pustejovsky pour les verbes causatifs formalise le célèbre kill $=$ cause become not alive (tuer $=$ causer devenir pas vivant) de (McCawley 1968). Un verbe causatif saturé dans une forme transitive $X V_{c} Y$ décrit un événement complexe, noté $\mathrm{e}_{0}$, décomposable en deux sous-événements notés $\mathrm{e}_{1}$ et $\mathrm{e}_{2}: \mathrm{e}_{1}$ est le sous-événement causal et e 2 le sous-événement correspondant à l'état résultant.

Quel que soit le verbe causatif, le constituant $Y$ doit référer à une entité y. L'état résultant $\mathrm{e}_{2}$ est l'état dénoté par le participe passé adjectival Vc-pp affectant y, si la morphologie le permet. Par exemple,

si $V c=$ énerver, alors $\mathrm{e}_{2}=\operatorname{ENERVE}(\mathrm{y})$

et si $V_{c}=$ fêler, alors $\mathrm{e}_{2}=\operatorname{FELE}(\mathrm{y})$,

mais si $V_{c}=$ tuer, alors $\mathrm{e}_{2}=\operatorname{MORT}(\mathrm{y})$ plutôt que TUE $(\mathrm{y})$.

Cet état résultant reflète que $X V_{c} Y$ peut grosso modo être paraphrasé par $X$ cause $Y$ être (devenir) $V c$ - $p$ p, si la morphologie le permet.

Pour un verbe causatif non psychologique comme fêler dont le sujet ne peut référer qu'à une entité (humaine), le «paradigme conceptuel lexical » du verbe est celui de la causation directe dans lequel le sous-événement causal dénote une action nonspécifiée de $\mathrm{x}$ sur $\mathrm{y}$, ce qui est noté $\mathrm{e}_{1}=$ ?ACTE $(\mathrm{x}, \mathrm{y})$.

La structure événementielle associée à un verbe comme fêler est schématisée dans la figure (4).

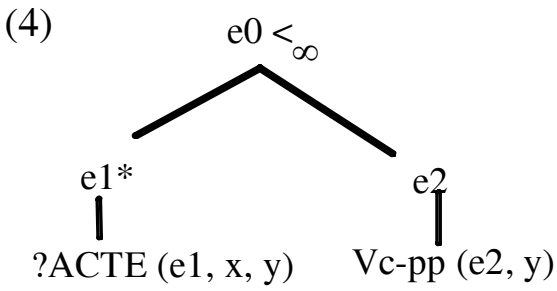

Nous renvoyons à (Pustejovsky 1995) pour les autres points de cette structure événementielle.

Pour un verbe causatif psychologique, l'entité y est basiquement humaine. Rappelons que le sujet $X$ dénote, soit un objet abstrait de type événement ou fait, soit une entité. Si $X$ dénote un objet abstrait, alors l'événement causal $\mathrm{e}_{1}$ est cet objet abstrait, ou plus précisément le fait que y perçoive ou ait conscience de cet objet abstrait : l'interprétation causale de (1a) implique que Marie ait entendu, vu ou appris que Fred avait heurté la carafe contre l'évier. Sinon, comment pourrait-elle en être agacée ? Si $X$ dénote une entité (humaine) x, alors x participe à un objet abstrait qui est l'événement causal $e_{1}$ (perçu physiquement ou intellectuellement par y). L'individu 
$x$ peut participer «activement» ou «passivement» à $\mathrm{e}_{1}^{6}$. Dans le cas d'une participation active, $\mathrm{x}$ est l'agent de l'action dénotée par $\mathrm{e}_{1}$. Dans le cas d'une participation passive, $\mathrm{x}$ est l'objet d'une prédication du type propriété. Ces différents cas sont analysés par coercion de type, analyse par laquelle un sujet dénotant une entité est dynamiquement coercé en un objet abstrait.

\subsection{Analyse par coréférence événementielle des discours causaux}

Muni de la décomposition lexicale des verbes causatifs proposée dans (Pustejovsky 1995), revenons aux discours causaux, en commençant par (2b-c) répétés ci-dessous en (5).

(5) a Fred a heurté la carafe contre l'évier. Il l'a fêlée.

b Fred a fêlé la carafe. Il l'a heurtée contre l'évier.

Notre analyse est la suivante : l'interprétation causale de ces discours vient du fait que le sous-événement causal du verbe causatif est interprété comme étant coréférent à l'événement décrit dans l'autre phrase (celle ne contenant pas le verbe causatif). En (5), on sait que l'action faite par Fred sur la carafe qui a résulté dans le fait qu'elle est fêlée est son heurtage contre l'évier. Les analyses de (5a) et (5b) sont schématisées dans les diagrammes ci-dessous (les symboles F, c et ev sont les constantes permettant de repérer les entités dénotées respectivement par Fred, la carafe et l'évier).

6a)

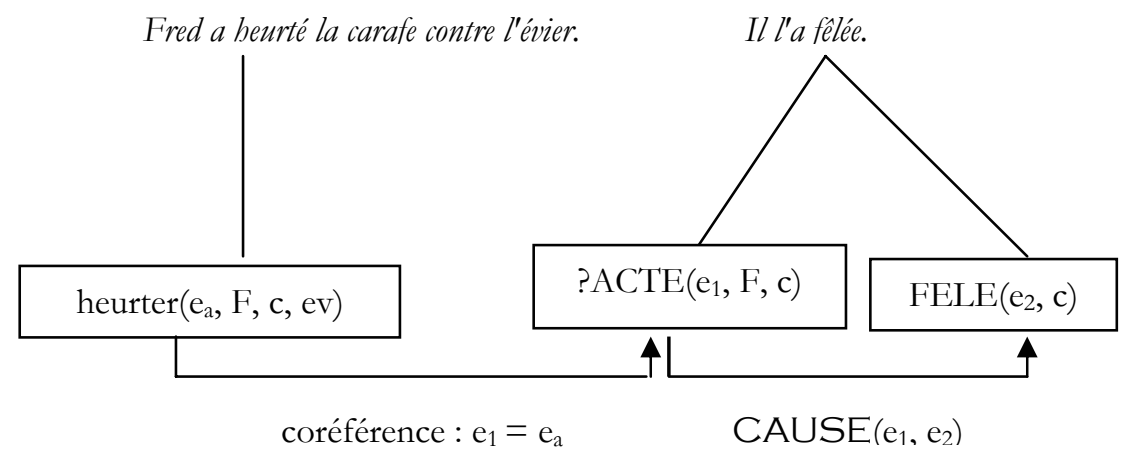

(6b)

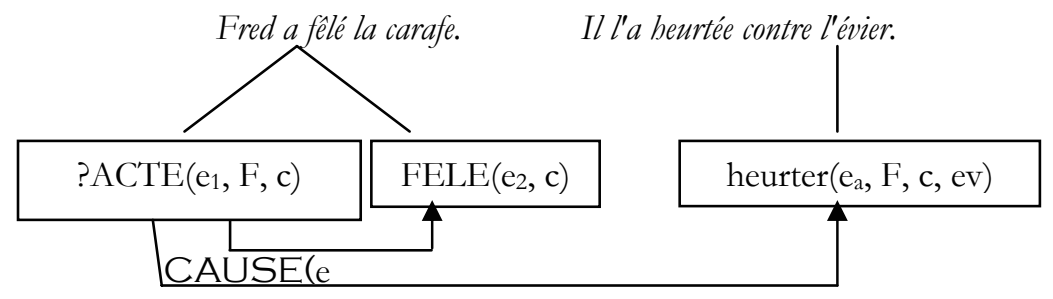

coréférence : $\mathrm{e}_{\mathrm{a}}=\mathrm{e}_{1}$

${ }^{6}$ Les termes de sujet actif et sujet passif sont employés dans (M. Gross 1975). 
Insistons bien sur le point suivant : pour les discours construits sur le modèle de (5), la relation de coréférence événementielle est une condition nécessaire et suffisante à une interprétation causale. Illustrons ce point sur les discours en (7).

(7) a Fred a photographié la carafe. Il l'a fêlée.

b Fred a fêlé la carafe. Il l'a photographiée.

D'aucuns y verront une interprétation causale, d'autres non. Peu importe l'interprétation, notre point est le suivant : les locuteurs qui donnent à (7a) ou (7b) une interprétation causale considèrent que l'acte de photographier la carafe est coréférent au sous-événement causal de fêler: l'action que Fred a faite qui a résulté en l'état fêlé de la carafe est le fait de l'avoir photographiée (par exemple, parce que Fred est muni d'une caméra digne de James Bond). Les locuteurs qui ne donnent pas une interprétation causale à (7) ne peuvent/veulent pas imaginer que photographier une carafe puisse induire un changement d'état pour la carafe, et par là-même excluent une relation de coréférence événementielle.

Nous voyons que la notion linguistique de "coréférence événementielle» permet de cerner les discours causaux qui nous intéressent, qui sont traditionnellement analysés comme exprimant une causalité directe (Fodor 1970, Schank 1975).

Cette analyse des discours causaux permet de préciser le point suivant sur les verbes causatifs non psychologiques. Si pour un bon nombre de verbes causatifs non psychologiques, le sous-événement causal est non spécifié, ce que nous traduisons par une variable de prédicat ?ACTE $\left(\mathrm{e}_{1}, \mathrm{x}, \mathrm{y}\right)$, certains de ces verbes ont un sousévénement causal spécifié et ils ne peuvent pas de ce fait apparaittre dans des discours de causalité directe. Considérons le verbe couper (une baguette). On peut préciser le résultat (Fred a coupé la baguette en fines tranches) ou l'instrument (Fred a coupé la baguette avec un bon couteau), mais on ne peut pas préciser le geste de Fred : celui-ci est déjà défini dans la sémantique du verbe couper. Autrement dit, le sous-événement causal de couper est totalement spécifié, ce que nous notons ACTE-COUPER( $\left(\mathrm{e}_{1}, \mathrm{x}, \mathrm{y}\right)$ sans variable de prédicat. Cette absence de variable explique qu'on ne peut pas faire précéder ou suivre une phrase $X$ couper $Y$ d'une phrase décrivant comment x s'y est pris?

Comme autres verbes causatifs dont le sous-événement causal est totalement spécifié, citons les verbes techniques tels que scier (une branche), goudronner (une route), cirer (un meuble), beurrer (une tartine) ou encore égorger, guillotiner, décapiter, électrocuter qui sont des troponymes de tuer et qui seront étudiés dans la Section 3.2. On notera que ces verbes n'admettent aucune forme inaccusative (pronominale ou pas), *la route a goudronné, *la route s'est goudronnée, alors que les verbes causatifs dont le sous-événement causal est non spécifié peuvent admettre une forme inaccusative, la carafe s'est fêlée, ? la carafe a fêlé,

\footnotetext{
${ }^{7}$ Nous ne pouvons pas prouver la non-existence de tels discours, néanmoins nous invitons le lecteur à essayer de construire de tels discours et à vérifier que ceci est impossible.
} 
la carafe s'est cassée, la carafe a casse $e^{8}$. On peut avancer l'hypothèse que les verbes causatifs à sous-événement causal spécifié n'admettent pas de forme inaccusative car une telle forme gommerait (passerait sous silence) une partie de la sémantique de ces verbes, à savoir l'acte causal. Néanmoins, cette hypothèse demande à être étayée par une vision exhaustive des données.

Pour les verbes causatifs psychologiques, rappelons que le sujet peut référer directement au sous-événement causal, voir (1a) répété en (8a), ou indirectement par métonymie, voir (1b) et (1c) répétés en (8b) et (8c).

(8) a Fred a heurté la carafe contre l'évier. Cela / Cet incident a agacé Marie.

b Fred a beurté la carafe contre l'évier. Il a agacé Marie.

c Fred a agacé Marie. Il a heurté la carafe contre l'évier.

Tous ces discours mettent en jeu une relation de coréférence événementielle. Il faudrait néanmoins rendre compte du fait que l'entité y (Marie dans nos exemples) doit avoir perçu ou appris la cause, mais nous n'entrerons pas ici dans ces détails?.

Tournons-nous maintenant vers les verbes causatifs dont le sous-événement causal est non-spécifié lorsqu'ils sont employés à la forme pronominale réfléchie, on a donc $\mathrm{x}=\mathrm{y}$. Dans le paradigme en (9), seuls les deux premiers exemples sont naturels et ont une interprétation causale (les deux derniers sont donc précédés du signe \#).

(9) a Fred s'est propulsé hors de la voiture. Il s'est blessé.

b Fred est tombé hors de la voiture. Il s'est blessé.

c \#Fred a été propulsé hors de la voiture. Il s'est blessé.

d \#Fred a reçu un pot de bégonias sur la tête. Il s'est blessé.

En nous appuyant sur les proto-rôles thématiques de (Dowty 1991), à savoir les notions de «Proto-Agent» et de «Proto-Patient», nous avons avancé dans (Danlos 1999) l'hypothèse (H1).

(H1) Dans un discours exprimant une relation causale directe, le résultat affectant une entité $X$ peut être construit autour d'un verbe causatif à la forme réfléchie si et seulement si $X$ est Proto-Agent dans la cause.

Ainsi Fred est Proto-Agent en (9a) et (9b) (même si la chute est involontaire) alors qu'il est Proto-Patient en (9c) et (9d). L'hypothèse (H1) permet aussi d'expliquer le contraste en (10) : (10a) est meilleur que (10b), ce qui va de pair avec le fait que le sujet de regarder est plus Proto-Agent que celui de voir.

(10) a Fred a regardé la foudre. Il s'est aveuglé.

b ? Fred a vu la foudre. Il s'est avenglé.

8 Toutefois, certains verbes causatifs dont le sous-événement causal est non-spécifié ne permettent pas de forme inaccusative, tuer et blesser par exemple.

9 Il faudrait aussi rendre compte du fait que la cause d'une changement d'état psychologique peut être un fait (et non un événement), comme en témoigne le discours (i) où la phrase décrivant la cause est négative : (i) Fred n'est pas venu. Cela a agacé Marie. 
Considérons aussi le contraste en (11) : on constate que la forme métonymique (i.e. Fred au lieu de la voiture de Fred) donne de l'agentivité à Fred et que de ce fait (11b) est meilleur que (11a).

\section{(11) a ? La voiture de Fred a percuté un camion. Il s'est blessé.}

b Fred a percuté un camion. Il s'est blessé.

Enfin, signalons que l'on peut se servir des discours causaux pour déterminer le proto-rôle thématique de certains arguments. Ainsi les discours acceptables en (12) tendent à indiquer que les sujets de éternuer et ronfler sont Proto-Agent alors que les tests proposé par (Dowty 1991) sont assez inopérants pour ces arguments.

(12) a Fred a éternué très fort. Il s'est fait mal aux côtes.

b Fred a ronflé toute la nuit. Il s'est irrité la gorge.

Dans cette section, aucune différence n'a été mise en avant selon que le verbe causatif apparait dans la première ou dans la seconde phrase des discours de causalité directe. La section suivante va se concentrer sur de telles différences.

\section{Typage des relations de coréférence}

\subsection{Définitions}

Commençons par définir deux types de relation de coréférence par rapport à la quantité d'informations apportée.

Deux descriptions successives D1 et D2 de la même entité x (événement ou entité) sont dans une relation de particularisation notée D2 = PART(D1) si et seulement si D2 apporte une ou des informations nouvelles sur x par rapport à D1.

Deux descriptions successives D1 et D2 de la même entité x (événement ou entité) sont dans une relation de généralisation notée D2 = GEN(D1) si et seulement si D2 n'apporte aucune information nouvelle sur $x$ par rapport à D1.

Illustrons ces deux types de coréférence sur le cas connu où x est une entité D1 et D2 sont alors des groupes (pro)nominaux. Dans (13a), le GN démonstratif cette Parisienne $=$ PART (une gynécologue) $\mathrm{car}$ il apporte l'information que le femme de Fred est parisienne. Dans (13b), le pronom elle = GEN (une gynécologue) car un pronom n'apporte aucune information nouvelle. Enfin, dans (13c) le GN défini ou démonstratif la/cette doctoresse $=\mathrm{GEN}$ (une gynécologue) du fait de la relation d'hyperonymie entre gynécologue et doctoresse (ce GN ne nous apprend pas que la femme de Fred est une doctoresse puisqu'on le sait du fait qu'elle soit gynécologue).

(13) a Fred s'est marié avec une gynécologue. Cette Parisienne agace Marie.

b Fred s'est marié avec une gynécologue. Elle agace Marie.

c Fred s'est marié avec une gynécologue. La/Cette doctoresse agace Marie. 
Quel type de coréférence est en jeu dans les discours de causalité directe ? Lorsque le verbe causatif apparait dans la seconde phrase, voir (5a) et son analyse en (6a), la coréférence événementielle $e_{1}=e_{a}$ est du type généralisation. En effet, la description de $\mathrm{e}_{1}$, à savoir ?ACTE $\left(\mathrm{e}_{1}, \mathrm{~F}, \mathrm{c}\right)$ n'apporte aucune information nouvelle par rapport à celle de $e_{\mathrm{a}}$, à savoir HEURTER $\left(\mathrm{e}_{\mathrm{a}}, \mathrm{F}, \mathrm{c}, \mathrm{ev}\right)$. On peut d'ailleurs considérer ?ACTE $\left(e_{1}, F, c\right)$ comme un hyperonyme de $\operatorname{HEURTER}\left(\mathrm{e}_{\mathrm{a}}, \mathrm{F}, \mathrm{c}, \mathrm{ev}\right)$. A l'inverse, lorsque le verbe causatif apparait dans la première phrase, voir (5b) et son analyse en (6b), la coréférence événementielle $\mathrm{e}_{\mathrm{a}}=\mathrm{e}_{1}$ est du type particularisation car la seconde description de l'événement concerné, à savoir la description de $e_{a}$, apporte de l'information par rapport à la première, à savoir celle de $\mathrm{e}_{1}$.

Cette différence dans le typage de la relation de coréférence événementielle va nous permettre d'expliquer les contrastes dans les discours de causalité directe selon l'ordre d'apparition des phrases.

\subsection{Particularisation et généralisation dans les discours causaux}

Nous allons examiner l'adjonction de modifieurs sur le verbe causatif dans nos discours causaux. Comme proposé dans (Pustejovsky 1991) et (Pustejovsky 1995), nous distinguons les modifieurs qui portent sur l'événement causal (et éventuellement sur l'état résultant), de ceux qui ne portent que sur l'état résultant.

Parmi les premiers se trouvent les modifieurs temporels (Fred a fêlé la carafe à midi implique que l'acte de Fred sur la carafe a eu lieu à midi), les modifieurs locatifs (Fred a fêlé la carafe dans la cuisine), et ceux qui sont orientés vers l'agent (Fred a cassé la carafe volontairement implique que Fred voulait que la carafe soit cassée lorsqu'il a fait une action dessus ${ }^{10}$ ).

Parmi les seconds se trouvent principalement les modifieurs indiquant un degré de l'état résultant : Fred a salement fêlé la carafe implique que la carafe est salement fêlée, autrement dit, l'adverbe salement ne porte que sur l'état résultant.

Commençons par les discours de causalité directe dans lesquels le verbe causatif apparait dans la première phrase. On constate que n'importe quel modifieur peut s'adjoindre sur le verbe causatif sans altérer l'interprétation causale, voir (14).

(14) a Fred a fêlé la carafe à midi. Il l'a heurtée contre l'évier.

b Fred a fêlé la carafe dans la cuisine. Il l'a beurtée contre l'évier.

c Fred a cassé la carafe volontairement. Il l'a heurtée contre l'évier.

d Fred a salement felé la carafe. Il l'a beurtée contre l'évier.

Par contre, lorsque le verbe causatif apparait dans la seconde phrase, l'adjonction de modifieurs portant sur l'événement causal altère l'interprétation causale: les discours en (15) ci-dessous ne peuvent pas recevoir d'interprétation causale, d'où le signe \#.

(15) a \# Fred a beurté la carafe contre l'évier. Il l'a fêlée à midi.

b \# Fred a heurté la carafe contre l'évier. Il l'a fêlée dans la cuisine.

c \# Fred a heurté la carafe contre l'évier. Il l'a cassée volontairement.

${ }^{10}$ Nous utilisons casser et non fêler car la phrase Fred a fêlé la carafe volontairement est déviante. 
Toutefois, l'adjonction d'un modifieur ne portant que sur l'état résultant n'altère pas l'interprétation causale, voir (15d).

\section{(15) d Fred a heurté la carafe contre l'évier. Il l'a salement fêlée.}

Comment expliquer le contraste entre les exemples en (14) et ceux en (15) ? Par le typage de la relation de coréférence événementielle avec l'hypothèse (H2).

(H2) Dans un discours exprimant une relation causale directe, la relation de coréférence événementielle doit être de type généralisation lorsque le verbe causatif apparaît dans la seconde phrase et de type particularisation lorsque le verbe causatif apparaît dans la première phrase.

Nous avons montré, à la fin de la section précédente, que l'hypothèse (H2) est respectée lorsque le verbe causatif n'est pas modifié par un adverbial, comme c'est le cas dans les exemples de base sous (5). Nous allons maintenant nous occuper des cas où le verbe causatif est modifié.

Commençons par expliquer comment (H2) rend compte du paradigme en (15) avec le verbe causatif dans la seconde phrase. En (15a), les informations sur le sousévénement causal $\mathrm{e}_{1}$ de fêler sont ?Acte $\left(\mathrm{e}_{1}, \mathrm{~F}, \mathrm{c}\right) \wedge$ à-midi $\left(\mathrm{e}_{1}\right)$. Or il n'y a aucune information temporelle sur l'événement $e_{a}$ de heurtage. La description de $e_{1}$ apporte donc de l'information nouvelle par rapport à celle de $\mathrm{e}_{\mathrm{a}}$. Par conséquent, la relation de coréférence événementielle n'est pas du type généralisation, ce qui explique d'après (H2) que (15a) ne reçoit pas d'interprétation causale. Même raisonnement pour (15b) et (15c). Par contre, en (15d), le modifieur salement porte sur $\mathrm{e}_{2}$ et non sur $\mathrm{e}_{1}$ : les informations concernant $\mathrm{e}_{2}$ sont $\mathrm{FELE}\left(\mathrm{e}_{2}, \mathrm{c}\right) \wedge$ méchamment $\left(\mathrm{e}_{2}\right)$, celles concernant $\mathrm{e}_{1}$ sont ?Acte $\left(e_{1}, F, c\right)$. La relation de coréférence événementielle entre $e_{1}$ et $e_{a}$ reste du type généralisation, ce qui explique d'après $(\mathrm{H} 2)$ que $(15 \mathrm{~d})$ garde une interprétation causale.

Passons au paradigme en (14) avec le verbe causatif dans la première phrase. En (14a), la description de $e_{a}$, qui spécifie l'acte de Fred sur la carafe, apporte de l'information nouvelle par rapport à celle de $\mathrm{e}_{1}$, même si elle ne comporte pas d'information temporelle. On comprend que le heurtage a eu lieu à midi, même si ce n'est pas dit explicitement mais implicitement via la relation de coréférence événementielle qui reste du type particularisation. D'après (H2), (14a) garde donc une interprétation causale. Même raisonnement pour (14b-d).

L'hypothèse (H2) permet d'expliquer d'autres contrastes dans les discours de causalité directe selon l'ordre des informations. Par exemple, le contraste en (16) ou $(17)^{11}$. Ces exemples montrent que les verbes causatifs assassiner ou se suicider peuvent

\footnotetext{
${ }^{11}$ Historiquement, j'ai mis en évidence le contraste entre (16) ou (17) au début des années 1980, dans mes travaux sur la Génération de Textes (Danlos 1985). N'ayant pas la moindre explication pour ce phénomène, je l'ai "codé en dur" dans mon générateur de dépêches journalistiques relatant des attentats. Ce n'est qu'à la fin des années 1990 que j'ai avancé l'hypothèse (H2) et les autres données présentées dans cet article. Je profite de cette occasion pour faire un petit coucou à mon exdoctorante et amie, Anne Guyon, laminée par un cancer en 1999. Anne, qui savait que je m'arrachais les cheveux sur les discours causaux, a retourné la côte de bœuf charollaise qui grillait sur le barbecue en me disant: "Tes discours causaux ne sont peut-être pas aussi symétriques que les deux faces de
} 
apparaitre dans la première phrase mais pas dans la seconde, alors qu'il n'y a aucune restriction pour les verbes twer ou se tuer.

(16) a Fred a assassiné / tué le Président de la République. Il lui a tiré dessus.

b \# Fred a tiré sur le Président de la République. Il l'a assassiné.

c Fred a tiré sur le Président de la République. Il l'a tué.

(17) a Fred s'est suicidé / tué. Il a sauté du 3ème étage.

b \# Fred a sauté du 3ème étage. Il s'est suicidé.

c Fred a sauté du 3ème étage. Il s'est tué.

Quelle est la différence sémantique entre assassiner et tuer ou entre se suicider et se tuer? Le verbe assassiner (resp. se suicider) signifie « donner la mort (resp. se donner la mort) avec l'intention de le faire », alors que le verbe tuer (resp. se tuer) n'a pas de trait intentionnel. Dans la décomposition lexicale de ces verbes causatifs, cette différence se traduit par le fait que la description du sous-événement causal de assassiner ou se suicider doit comporter l'information que l'acte de $\mathrm{x}$ sur $\mathrm{y}$ (avec éventuellement $\mathrm{x}=\mathrm{y}$ ) est accompli avec l'intention d'atteindre l'état résultant $\operatorname{MORT}\left(\mathrm{e}_{2}, \mathrm{y}\right)$, alors qu'aucune information sur l'intention de $\mathrm{x}$ ne doit figurer dans la description du sous-événement causal de tuer ou se tuer.

Examinons les répercussions de cette différence de sémantique lexicale dans les discours (16) ou (17), en commençant par expliquer comment l'hypothèse (H2) rend compte de l'inacceptabilité - la non-interprétation causale - de (16b) ou (17b). La seconde phrase de ces discours - construite autour de assassiner ou se suicider comporte l'information sur l'intention de x. Or cette information n'est pas présente dans la première phrase de (16b) ou (17b) qui décrit une action et non l'objectif de cette action ; par exemple, la première phrase de (17b) peut correspondre à une situation où Fred a sauté du troisième étage dans le but d'épater Marie et pas du tout dans celui de se tuer. La relation de coréférence événementielle n'est donc pas du type généralisation et ces discours n'ont pas d'interprétation causale d'après (H2).

Par contre, en (16c) ou (17c), la description des sous-événements causaux des verbes causatifs - tuer ou se tuer - ne comportant pas de trait intentionnel, la relation de coréférence événementielle reste du type généralisation, et ces discours ont une interprétation causale d'après $(\mathrm{H} 2)$.

Pour (16a) ou (17a), la seconde phrase apporte de l'information nouvelle par rapport à ce qui est connu sur le sous-événement causal du verbe causatif, que celui-ci porte ou non un trait intentionnel. La relation de coréférence événementielle est du type particularisation, et ces discours ont une interprétation causale d'après ( $\mathrm{H} 2)$.

L'hypothèse (H2) permet aussi d'expliquer le contraste en (18) où les premières phrases sont au passif sans agent. Le discours (18a) ne reçoit pas d'interprétation causale d'après (H2) car la description du sous-événement causal $e_{1}$ apporte l'information que l'agent de l'acte sur la carafe est Fred, information absente de la première phrase au passif sans agent : la relation de coréférence événementielle n'est

cette côte de bœuf". Ses propos furent le déclic qui me conduisit à postuler la dissymétrie présente dans l'hypothèse (H2). 
pas du type généralisation. Par contre, l'interprétation causale de (18b) ne pose pas de problème car la relation de coréférence événementielle est bien du type particularisation.

\section{(18) a \# La carafe a été heurtée contre l'évier. Fred l'a fêlée. \\ b La carafe a été fêlée. Fred l'a beurtée la carafe contre l'évier.}

Enfin, nous allons terminer cette section en examinant les troponymes des verbes causatifs. Rappelons que la notion de «troponyme » pour les verbes, introduite dans WordNet (Fellbaum 1998), peut être considérée comme l'équivalent de la notion d'« hyponymie » pour les noms : un verbe $V_{i}$ est un troponyme d'un verbe plus général $\mathrm{V}_{0}$ si $\mathrm{V}_{\mathrm{i}}$ " précise la façon dont $\mathrm{V}_{0}$ est réalisé ». Parmi les verbes non causatifs gifler est un troponyme de frapper, siroter (du pastis) un troponyme de boire (du pastis). Parmi les verbes causatifs, égorger, guillotiner, décapiter, électrocuter sont des troponymes de tuer. Quelle est la décomposition lexicale d'un verbe comme égorger ? Son sous-événement causal est totalement spécifié et ne contient clairement aucune variable de prédicat, autrement dit on a ACTE-EGORGER( $\left(\mathrm{e}_{1}, \mathrm{x}, \mathrm{y}\right)$ - rappelons qu'à l'inverse le sousévénement causal de tuer est non spécifié. L'état résultant de égorger est EGORGE(e, y), où le participe passé égorgé peut être considéré comme un hyponyme (troponyme ?) de mort.

Examinons maintenant les discours comprenant deux phrases, l'une construite autour d'un verbe causatif, l'autre autour d'un troponyme de ce verbe causatif, les arguments étant sémantiquement identiques. Commençons par le cas où le troponyme se trouve dans la seconde phrase, cas illustré en (19).

Fred a tué un lapin. Il l'a égorgé.

Le discours (19), parfaitement naturel, peut à première vue être qualifié de discours à causalité directe, dans la mesure où la seconde phrase spécifie l'acte accompli par Fred pour tuer le lapin. Mais regardons de plus près. Chaque verbe causatif dénote un événement complexe, $\mathrm{e}_{0}$ pour tuer et $\mathrm{e}_{0}^{\prime}$ pour égorger, décomposable en un sous-événement causal, $\mathrm{e}_{1}$ pour tuer et $\mathrm{e}_{1}$ pour égorger, et un état résultant, $\mathrm{e}_{2}$ pour tuer et e'2 pour égorger. Si on considère que (19) est un discours de causalité directe, cela implique, d'après notre analyse, que l'événement décrit dans la seconde phrase, soit $\mathrm{e}_{0}^{\prime}$, est coréférent au sous-événement causal de tuer, soit $\mathrm{e}_{1}$. Mais cette relation de coréférence ne fait pas sens : c'est e'1 qui est coréférent à e $e_{1}$. Le discours (19) n'est donc pas un discours de causalité directe. Par contre, il est tout à fait justifié de poser que $\mathrm{e}_{0}$ et $\mathrm{e}_{0}^{\prime}$ sont coréférents, autrement dit que (19) est un discours enchainant deux phrases référant au même événement, la relation de coréférence étant de type particularisation puisque la seconde phrase apporte de l'information nouvelle par rapport à la première. Nous avons étudié en détail ce type de discours dans (Danlos 2001b) ou (Danlos $2004)^{12}$. En résumé, un discours comme (19) met en jeu trois relations de coréférence

12 Un autre exemple de ce type de discours est donné en (i). Les deux phrases décrivent le même événement, la seconde phrase apportant de l'information nouvelle par le biais de lapin, hyponyme de animal: (i) Fred a égorgé un animal. Il a égorgé un lapin.

On notera que les deux groupes nominaux indéfinis un animal et un lapin sont coréférents, ce qui contredit l'hypothèse généralement admise qu'un GN indéfini introduit un nouveau référent de 
événementielle, à savoir $\mathrm{e}_{0}^{\prime}=\mathrm{e}_{0}, \mathrm{e}_{1}^{\prime}=\mathrm{e}_{1}$ et $\mathrm{e}_{2}^{\prime}=\mathrm{e}_{2}$, ces trois relations étant du type particularisation.

Passons maintenant au cas où le troponyme apparait dans la première phrase, cas illustré en (20).

(20) a * Fred a égorgé un lapin. Il l'a tué.

b Fred a égorgé un lapin. Il l'a tué à midi.

Le discours (20a) est perçu comme non naturel car la seconde phrase est totalement redondante par rapport à la première, elle n'apporte aucune information. Par contre, (20b) est naturel car la seconde phrase apporte une information temporelle par rapport à la première. Ces discours sont à rapprocher des discours en (21) ou (22).

(21) a * Fred a égorgé un lapin. Il a fait cela.

b Fred a égorgé un lapin. Il a fait cela à midi.

(22) a * Fred a giflé Marie. Il l'a frappée.

b Fred a giflé Marie. Il l'a frappée à midi.

Les discours en (21) mettent en jeu l'anaphore verbale faire cela, ceux en (22) mettent en jeu la paire gifler-frapper où le premier verbe est un troponyme du second (sans que ces verbes soient causatifs). Dans tous les discours (20b), (21b) et (22b), les deux phrases réfèrent au même événement, la seconde phrase apportant une information nouvelle (temporelle) par rapport à la première.

En résumé, les discours (19) et (20) comportant un verbe causatif et un troponyme de ce verbe causatif ne sont pas des discours exprimant une causalité directe mais des discours enchaînant deux phrases référant au même événement. Les acceptabilités en (20), qui sont exactement l'inverse de ce que prédirait (H2), ne sont pas des contre-exemples à l'hypothèse $(\mathrm{H} 2)$ puisqu'il n'y a pas de relation de causalité directe.

\section{Conclusion}

En nous appuyant sur la décomposition lexicale des verbes causatifs proposée dans (Pustejovsky 1995), nous avons montré que les discours exprimant une causalité directe mettaient en jeu une relation de coréférence événementielle entre l'événement décrit dans la cause et le sous-événement causal du verbe causatif.

Un typage des relations de coréférence selon l'apport d'information nous a permis de mettre en évidence et d'expliquer différents contrastes selon que le verbe causatif apparait dans la première phrase ou dans la seconde.

Les exemples présentés dans cet article enchainent deux phrases indépendantes non reliées par un connecteur de discours. Le lecteur vérifiera que les données sur la causalité directe ne changent pas si l'on utilise un participe présent ou un gérondif comme illustré en (23).

discours. Pour sauver cette hypothèse, nous proposons dans (Danlos et Gaiffe 2004) de calculer cette relation de coréférence inédite par le biais d'une nouvelle relation de discours entre les deux phrases. 
Verbes causatifs, discours causaux et coréférence événementielle

(23) a Fred a heurté la carafe contre l'évier la fêlant.

b Fred a fêlé la carafe en la heurtant contre l'évier.

Notre analyse par une relation de coréférence événementielle typée généralisation ou particularisation fonctionne pour (23) comme pour les discours parataxiques.

Dans cet article, nous avons fait abstraction de la notion de «relation de discours ", qui est centrale dans toute étude sur le discours. Néanmoins, dans d'autres travaux, nous avons prolongé les données et analyses présentées ici par l'étude des relations de discours en jeu, voir (Danlos et Gaiffe 2004) et (Danlos 2006). Ces études montrent que les données de sémantique lexicale enrichissent les recherches sur le discours et vice-versa. 


\section{RÉFÉRENCES BIBLIOGRAPHIQUES}

DANLOS, L., 1985, Génération automatique de textes, Masson.

DANlos, L., 1999, "Discours causal et rôles thématiques », in M. Plénat (ed) Des structures linguistiques à leur interprétation, mélanges en l'honneur d'André Borillo. Rodopi : 83-99.

DANLOS, L., 2001a, "Événement Coreference in Causal Discourses », in P. Bouillon et F. Busa, (eds), Meaning of Word, Cambridge University Press : 216-241.

Danlos, L. 2001b, «Event Coreference Between Two Sentences », in H. Bunt, R. Muskens and E.Thijsse (eds), Computing Meaning Volume 2, Kluwer Academic Publishers, Amsterdam : 271-288.

DANLOS, L., 2004, « Coréférence événementielle entre deux phrases », in E. Laporte, C. Leclère, M. Piot et M. Silberztein (eds.), Syntaxe, Lexique et Lexique-Grammaire. Volume dédié à Maurice Gross, Lingvisticae Investigationes Supplementa 24., John Benjamins Publishing Co.

Danlos, L., Gaiffe, B., 2004, «Event coference and discourse relations », in L. Kulda (éd), Language, Music and Cognition, Kluwer Academic Publishers, Amsterdam.

DANLOS, L., 2006, "Discourse verbs and discourse periphrastic links », in Proceedings of the Workshop on Constraints in Discourses, Dublin : 59-65.

DowTy, D, 1991, «Thematic Proto-Roles and Argument Selection », Language, vol 67 n 3.

FODOR, J., 1970, «Three reasons for Not Deriving kill from cause to die », Linguistic Inquiry : 429-438.

Fellbaum, C., A Semantic Network of English Verbs, WordNet an Electronic Lexical Database, MIT Press, 1998 : 69-103.

Gross, M., 1975, Méthodes en syntaxe, Hermann.

MCCAwley, J., 1968, «Lexical Insertion in a Transformational Grammar without Deep Structure », B. Barden, C-J. Bailey and A. Davidson (eds), Papers from the fourth Regional Meeting of the Chicago Linguistic Society, Chicago.

Pustejovsky, J., 1991, «The syntax of event structure », in B. Levin and S. Pinker (eds), Lexical and Conceptual Semantics, Elsevier Science Publishers, Amsterdam.

Pustejovsky, J., 1995, The generative Lexicon, The MIT Press.

SCHANK, R.,1975, Conceptual Information Processing, Amsterdam : North Holland. 